\title{
Peripheral Nerve Transplantation: The Effects of Predegenerated Grafts and Immunosuppression
}

\author{
Thomas E. Trumble \\ Department of Orthopaedics RK-10, University of Washington \\ 1959 N.E. Pacific Street, Seattle, Washington 98195, U.S.A.
}

\begin{abstract}
SUMMARY
Research involving nerve transplantation has shown that tissue rejection limits the neurologic recovery unless the host is immunosuppressed. This study investigates an alternative to permanent or temporary immunosuppression using a rat model with nerve transplants from BrownNorway rat donors to bridge defects in the sciatic nerve of Lewis rat recipients as these two inbred strains differ at both major and minor histocompatibility loci. The specific aim of this study was to evaluate if predegenerated nerve grafts decreased the tissue rejection and improved the neurologic recovery of animals with allogenic nerve grafts to avoid the problems associated with either short- or long-term immunosuppression. The animals in the experimental groups received cyclosporin-A, predegenerated grafts, both, or neither. The predegenerated grafts were produced by division of the nerve three weeks prior to grafting to allow for Wallerian degeneration to occur. The outcome was assessed by measurements stressing functional recovery (sensory testing, gait analysis, joint flexion contracture), studies of muscle recovery (muscle weight and hydroxyproline concentration), and histologic studies (axonal counts and inflammatory reaction). The animals receiving the predegenerated grafts without cyclosporin did have an improved recovery (joint flexion contracture $35^{\circ} \pm 8^{\circ}$ and hydroxyproline ratio $1.52 \pm 0.16$ ) as compared to the joint flex-
\end{abstract}

ion contractures and hydroxyproline ratios of the allograft group of animals without either cyclosporin-A or pretreatment and the ungrafted control group $\left(47^{\circ} \pm 18^{\circ}, 1.68 \pm 0.34\right.$, and $53^{\circ}$ $\pm 15^{\circ}, 4.50 \pm 0.27$, respectively, $p<0.01$ ). However, all the isograft groups and allograft groups with cyclosporin-A, regardless of whether the graft had been predegenerated or not, had greater neurologic recovery than the allograft group with predegenerated grafts but without cyclosporin-A by the same parameters ( $p<$ 0.01). Allograft groups with short-term immunosuppression with cyclosporin-A did as well as isograft groups, and isograft groups with predegenerated grafts did not do any better than isografts without pretreatment $(p<0.01)$.

Clinical Relevance: Predegenerated nerve allografts will allow for greater neurologic recovery than standard nerve allografts avoiding the complications of immunosuppression, but the level of recovery is less than that of recipients of nerve allografts with immunosuppression. Nerve transplants would avoid the problems of neurologic deficits at the donor site and allow multiple large deficits to be treated easily.

\section{KEY WORDS}

nerve, transplant, immunology, allograft, Wallerian degeneration 


\section{INTRODUCTION}

Currently, peripheral nerve injuries are treated by autogenous nerve grafts frequently obtained from areas such as the sural nerve. Many techniques have been explored to overcome these segmental deficits in peripheral nerves without requiring the use of autogenous grafts. With autogenous grafts, there is an obligatory neurologic deficit at the donor site plus the problem of a limited supply of graft material when treating large peripheral nerve injuries. Despite techniques using synthetic or fibrous sheath grafts, there is limited regeneration for segmental gaps in nerves greater than one centimeter. Thus, nerve cable grafts are still the most effective way to treat segmental injuries in peripheral nerves. Allogeneic transplantation of peripheral nerves would overcome these problems; however, neurologic recovery with allogeneic grafts has been limited by tissue rejection /1-4/. Early efforts to avoid the rejection phenomena have centered on decreasing the immunogenicity of the graft or attenuating the host response to the graft /4-8/. The Schwann cells are the immunogenic component of the nerve /19/. Freezing the grafts or treating them with high doses of radiation does limit the rejection response to nerve allografts. However, this technique eliminates the viable Schwann cells and drastically reduces the neurologic recovery when these tissues are used for grafting /6$8,10,11,20 \%$. Host immunosuppression has been accomplished using chemotherapy using corticosteroids, azathioprine, and cyclosporin-A, either with short-term or long-term treatment 17,9,11-16/. However, even short-term immunosuppression presents a serious risk to the host in terms of hepatic and renal toxicity as well as limiting the overall neurologic recovery. This study investigates graft pre-treatment using nerve graft segments that have undergone Wallerian degeneration as a means of improving the neurologic recovery of animals following transplantation without the need for immunosuppression. During Wallerian degeneration the Schwann cells are transformed to phagocytic cells that may have decreased expression of antigenic markers. This study stresses the functional recovery of the animal, as techniques which primarily emphasize histologic or electrophysiologic parameters do not correlate with parameters that are useful clinically $/ 8,9,18-26 /$.

\section{MATERIALS AND METHODS}

\section{Animal model}

Adult male rats were used in order to minimize the possibility of hormonal changes during the experiments that could affect results of transplantation. Brown-Norway rats (250-300 g, Harlan Sprague-Dawley, Indianapolis, IN) served as the donors and Lewis rats (300-350 g, Charles River Breeding Laboratory, Bedford, MA) served as the recipients and donors. These strains have been shown to differ in both major and minor histocompatibility loci $/ 27 /$. The rats were maintained in separate facilities in order to prevent viral contamination as there is a significant degree of microbial incompatibility between the two strains. The animals were assigned to one of eight experimental groups ( $n=15$ for each group; Table 1). Dependent on the group that they were assigned to, the animals in Groups A through $G$ received nerve grafts either with or without pretreatment by Wallerian degeneration, temporary host immunosuppression, both or neither. The Group $\mathrm{H}$ was left ungrafted as a control after a $2.0 \mathrm{~cm}$ defect was made in the sciatic nerve.

TABLE 1

Transplantation protocol

\begin{tabular}{lcccc}
\hline Group Recipient & Donor & $\begin{array}{c}\text { Cyclo- } \\
\text { sporin-A }\end{array}$ & $\begin{array}{c}\text { Wallerian } \\
\text { Degeneration }\end{array}$ \\
\hline A & Lewis & Lewis & - & 0 \\
B & Lewis & Lewis & + & 0 \\
C & Lewis & Lewis & - & $*$ \\
D & Lewis & Brown-Norway & - & 0 \\
E & Lewis & Brown-Norway & + & 0 \\
F & Lewis & Brown-Norway & - & $*$ \\
G & Lewis & Brown-Norway & + & $*$ \\
H & Lewis & Gap & None & None \\
\hline
\end{tabular}

\footnotetext{
- $=$ no cyclosporin treatment given

$+=$ cyclosporin treatment given

$0=$ no predegeneration of the graft

* = graft pretreated with Wallerian degeneration
} 
Predegenerated grafts were obtained by division of the sciatic nerve approximately three weeks prior to the final graft harvest. The animals were anesthetized with $30-50 \mathrm{mg} / \mathrm{kg}$ of pentobarbital sodium (Abbott Laboratories, Quebec, Canada) administered intraperitoneally. Under aseptic technique, a gluteal muscle splitting incision was made to expose the sciatic nerve. The nerve was then divided just as it exits from the sciatic notch. The wound was then closed in two layers with adsorbable sutures. After three weeks, a $2.5 \mathrm{~cm}$ segment was removed for nerve grafting using the same surgical technique to place a $2.5 \mathrm{~cm}$ graft into a 2.0 $\mathrm{cm}$. defect coapting the ends using standard microsurgical technique with $10-\mathrm{O}$ nylon.

The animals in Groups B, E and $G$ received cyclosporin-A orally in an olive oil suspension (Sandoz Inc., East Hanover, NJ) delivered by gavage $(10 \mathrm{mg} / \mathrm{kg} /$ day $)$ for twelve weeks. The animals were then maintained for another four weeks prior to sacrifice as rejection of peripheral nerves occurs within three weeks in the absence of immunosuppression or host incorporation $/ 12,14 /$. This dose of cyclosporin has been demonstrated to be effective without causing significant toxicity /28/. Animals in the control groups received olive oil alone. At the conclusion of the 16 weeks, the animals were sacrificed after the final testing was performed in order to remove the nerve graft along with $5 \mathrm{~mm}$ of the host nerve on either end, the contralateral nerve, the tibialis anterior muscle from the operated limb and the tibialis anterior muscle from the non-operated limb.

\section{Sensory testing}

The animals' response to light touch pressure and pin prick were recorded as follows: Grade 0 (no response), Grade 1 (withdrawal behind limb less than $1.0 \mathrm{~cm}$ ), and Grade 2 (withdrawal more than $1.0 \mathrm{~cm})$. The animals were tested before and after the sciatic nerve was blocked with $1 \%$ lidocaine or the animals were lightly anesthetized using $10-20 \mathrm{mg} / \mathrm{kg}$ of pentobarbital intraperitoneally. Light touch was assessed with Semmes-Weinstein filaments. Pressure was determined using a clamp calibrated at $30 \mathrm{~g} / \mathrm{cm}^{2}$.

\section{Gait testing and flexion contracture assessment}

The sciatic function index (SFI) described by deMedinaceli et al. /29/ was carried out on all animals. This is a combined scoring system that applies a formula using measurements from pawprints of toe spread and stride length. The toe spread has been noted to be the most reliable indicator in the gait analysis $/ 30 \%$ As in a previous study, the animals in the experimental groups were noted to have insufficient recovery of foot intrinsic muscles to allow for reproducible gait patterns within the experimental groups. Therefore, a technique of assessing functional recovery through the measurement of flexion contracture of the hind limbs was incorporated /31/. Under light general anesthesia, passive motion in the operated and unoperated leg was determined by suspending a $10 \mathrm{~g}$ weight from the foot and measuring the degree of ankle extension with a goniometer. This weight was sufficient to elicit the maximum joint extension. Control animals with only the muscle splitting incision did not develop any joint contracture. The degree of flexion contracture was defined as the difference between the passive extension of the normal and operated sides.

Tibialis anterior muscle weight and hydroxyproline assay

The tibialis anterior (TA) muscle was harvested from both hind limbs at the time of sacrifice. The muscle was removed in its entirety and weighed to the nearest $0.0001 \mathrm{~g}$ (Sartorius Analytic Balance Model 2603, Munich, West Germany). The muscle weight data were reported as a ratio of the operated leg to that of the unoperated limb.

The muscle hydroxyproline content was determined using the technique described by Woessner /32/. Muscle samples were hydrolyzed, oxidized using chloramine $\mathrm{T}$ (Fisher 01770, Reagent Grade, Fisher Chemicals, Kent, Washington) and the color was analyzed spectrophotometrically (LKB Biochron Ultraspec 4050, $\mathrm{AB}$ Bromma, Sweden), following the addition of the developer, dimethylaminebenzaldehyde, and using an adsorbency of $561 \mathrm{~nm}$. Distribution of hydroproline was restricted to the scleroproteins, collagen and elastin, pattern of muscle fibrosis 
which increases the content of the scleroproteins in muscle $/ 33 /$. The results were reported as the ratio of hydroxyproline concentration in the muscle of the operated side to that of the unoperated control limb.

\section{Histologic studies}

Four months following the nerve grafting, the animals were re-explored in order to harvest the graft segments along with portions of the host nerve and the contralateral nerve. The segments were immersed in Karnovsky's fixative, washed in cacodilated buffer, post-fixed with $1 \%$ osmium tetraoxide, dehydrated in graded alcohols, and embedded in epon. Sections one micron in thickness were prepared using an ultramicrotome (LKB Huxley, AB Bromma, Sweden) with a glass knife. Axon counts were performed using a Zeiss Videoplan (Munich, West Germany) with custom software for the calculation of area and point counting. The results were expressed as the ratio of the density $\left(\mathrm{axon} / \mathrm{mm}^{2}\right)$ of the fibers and the graft to the density in contralateral nerve. The Videoplan was also used to assess the relative histologic specimens that had infiltration by inflammatory cells on cross-sections from the mid point of the graft.

\section{Serum cyclosporin-A assay}

Serum cyclosporin-A levels were determined four weeks following the onset of the immunosuppressant therapy using a commercially available immunoassay for cyclosporin-A (CycloTrac, Incstar Corporation, Stillwater, MN). This technique is based on an equilibration procedure using double antibody phase separation and an iodine $^{125}$ tracer. The parent molecule and its nine biologically active metabolites are measured by this technique.

\section{Statistical analysis}

An analysis of variance was conducted within and among different experimental groups by means of the Kruskal-Wallis ANOVA nonparametric test. Individual comparisons in the groups were tested for probabilities and levels of significance by means of the Dunn analysis for multiple comparisons using rank sums and
Student's t-test where appropriate.

\section{RESULTS}

\section{Sensory testing}

Repeated testing with pressure pin-prick and light touch did not reveal any consistency even within experimental groups. Even groups having reinnervation as assessed by the other parameters had inconsistent results with the sensory tests. Even the gap control animals demonstrated some withdrawal response. Anatomical studies have shown that crossover innervation from the saphenous nerve is a predictable phenomenon in rats after sciatic nerve injury /34/. Our results seemed consistent with this as there were occasional examples of toe loss due to self-mutilation and this was always the most lateral toe, which is farthest from the distribution of the saphenous nerve.

\section{Gait analysis and flexion contracture}

As mentioned previously, the usefulness of the gait analysis was questioned early in the experiment when there was noted to be a lack of return in the intrinsic muscles to the foot in all grafted groups. Even after trying to modify the SFI formula to emphasize either stride length or toe spread, the gait analysis still did not provide reproducible results within single groups. In contrast to the use of the SFI when there is a lesion in continuity $/ 29,35 /$, efforts to base the SFI on the stride length were hampered by the fact that this parameter seems to be most heavily influenced by the animal's motivation.

Measurements of the flexion contracture were reproducible within experimental groups (Fig. 1). There was no significant difference between any of the isografted groups or the allografted groups which received cyclosporin-A. Furthermore, there was no significant difference between the allografted group which received both cyclosporin-A and pretreated grafts (Group $\mathrm{G})$ versus the group with immunosuppression but without the pretreated graft (Group E). The group with the pretreated graft but no host immunosuppression (Group F) had smaller flexion contractures $\left(35^{\circ} \pm 8^{\circ}\right)$ when compared 


\section{KEY TO FIGURES:}

Iso $=$ isogenic grafts

Iso/CyA = isogenic grafts and host immunosuppression with cyclosporin-A

Iso/Wall = isogenic grafts that underwent Wallerian predegeneration

Allo $=$ allogenic grafts without pretreatment or cyclosporin-A immunosuppression

Allo/CyA = allogenic grafts and host immunosuppression with cyclosporin-A

AlloWall = allogenic grafts that underwent Wallerian predegeneration

Allo/CyAWall = allogenic grafts that underwent Wallerian predegeneration and host immunosuppression with cyclosporin-A

Gap $=$ control animals that were not grafted.

FIGURE 1 - FLEXION CONTRACTURES

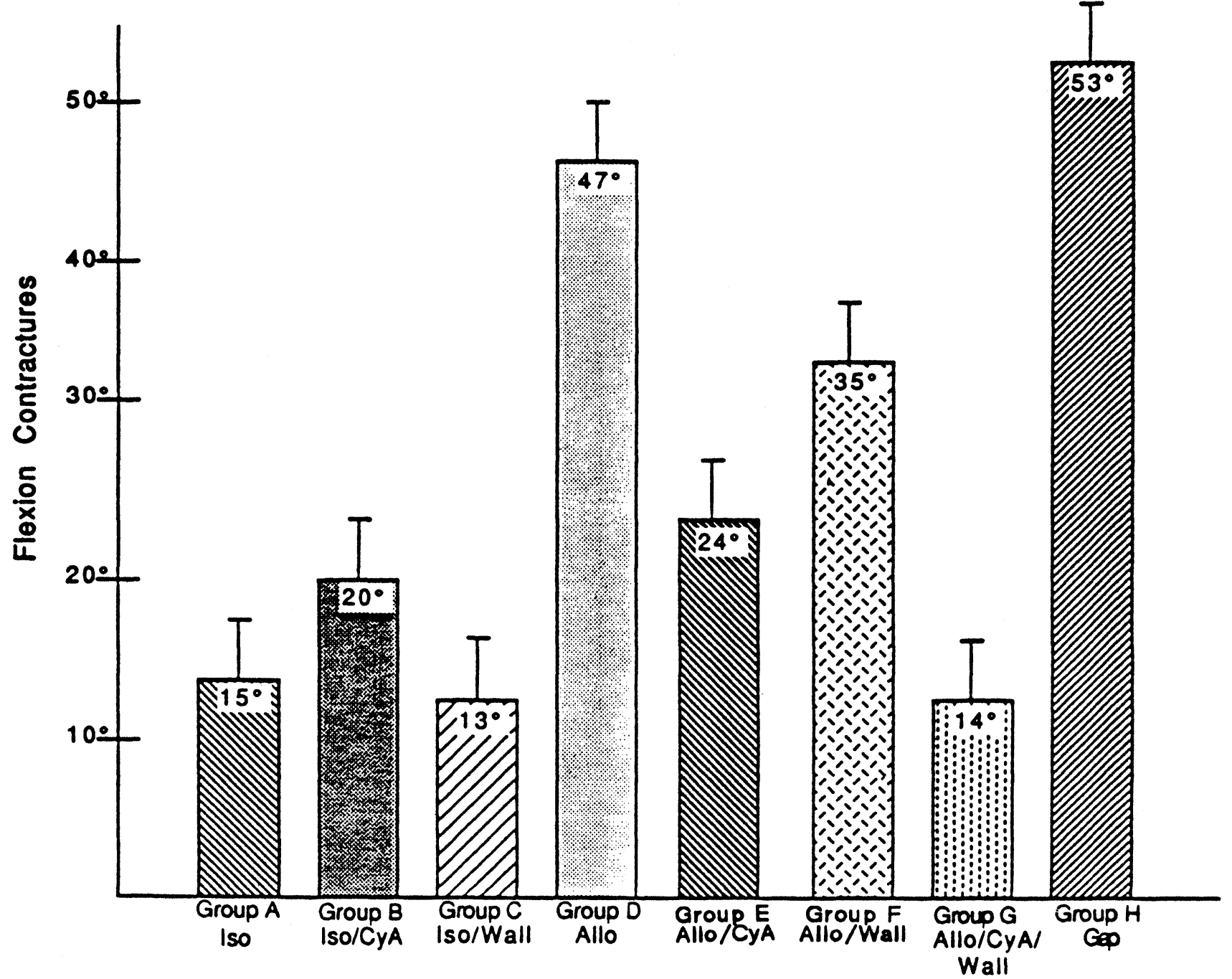

Fig. 1: The hind limb flexion contracture in degrees is obtained by subtracting the total passive extension of the operated limb from that of the unoperated limb. 
to the allografted group without pretreated grafts (Group $\mathrm{D}, 47^{\circ} \pm 10^{\circ}$ ) or the ungrafted gap control group (Group $\mathrm{H}, 53^{\circ} \pm 15^{\circ}$, p < 0.01). However, the flexion contractures in Group $F$ were significantly larger than in the remaining groups which had either isografts or allografts with immunosuppression $(p<0.01)$.
Tibialis anterior muscle weight and hydroxproline assay

The results are reported as the ratio of the tibialis anterior muscle weight in the operated limb compared to the non-operated limb (Fig. 2 ). There were no significant differences in these

\section{FIGURE 2 - MUSCLE WEIGHT}

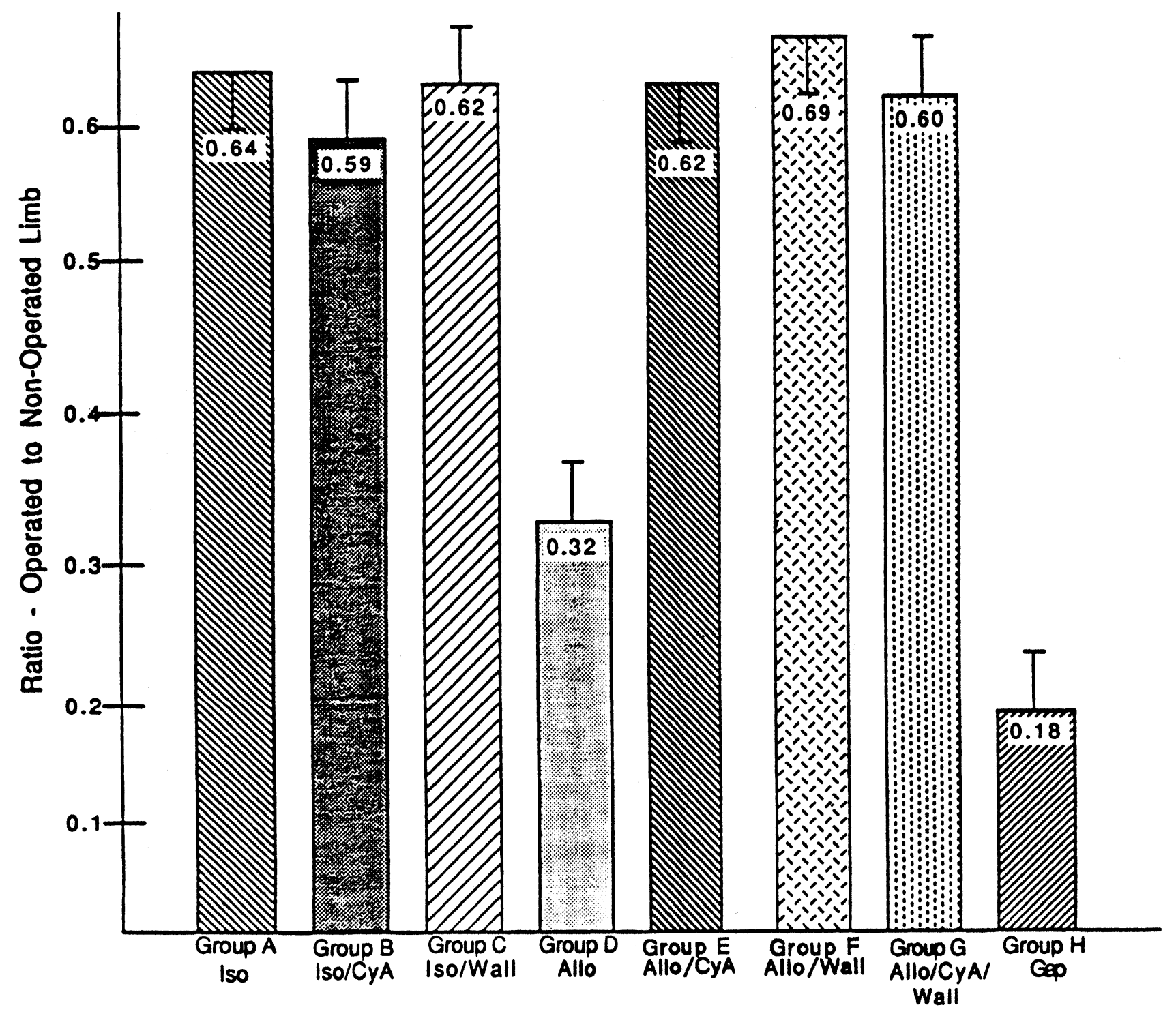

Fig. 2: Muscle weight. The muscle weight of the tibialis anterior muscle is expressed as the ratio of the operated tibialis anterior muscle weight compared to the non-operated control limb. 
ratios between any of the grafted groups; however, the gap control group was significantly lower than the grafted groups $(0.18 \pm 0.09, \mathrm{p}<$ $0.01)$. The pattern for the hydroxyproline concentration (Fig. 3) was similar to the results of the flexion contracture in that the group with pretreated allografts but no immunosuppression (Group F) had a lower hydroxyproline concentration $(1.52 \pm 0.16)$ than the allografted group with no pretreatment or immunosuppression $(1.68 \pm 0.34$, Group D) or the ungrafted group (Group H, $4.50 \pm 0.27$, p $<0.01$ ), but a higher hydroxyproline concentration than the groups with either isografts or allografts with immunosuppression (Groups A, B, C, E and G, p < $0.01)$. Similarly, there were no significant differences between the isografted groups or the allografted groups with immunosuppression. There was no significant difference between the allografted group that received immunosuppression plus a pretreated graft (Group $G$ ) versus Group $E$ which received an allograft with only immunosuppression of the host.

\section{FIGURE 3 - HYDROXYPROLINE CONCENTRATION}

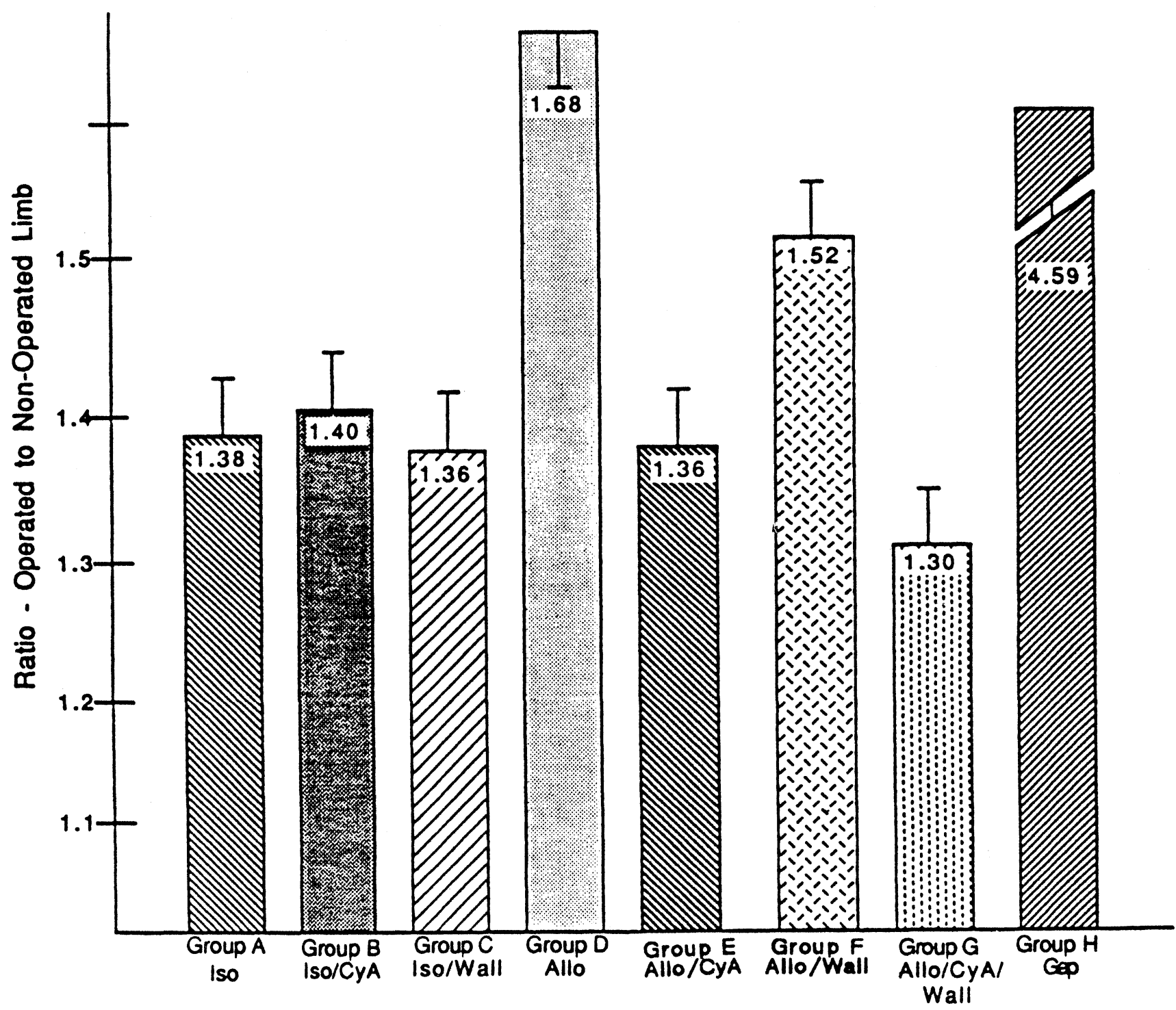

Fig. 3: Hydroxyproline concentration. The results are expressed as the ratio of the hydroxyproline concentration in the operated limb tibialis anterior muscle compared to the non-operated control limb. 


\section{Histologic studies}

All grafted groups showed evidence of axonal ingrowth both into the graft and across the distal coaptation site. In general, the axons in the graft were smaller, more variable in size, more widely spaced, and greater in number than those in the contralateral nerve. There was wide variability in the degree of myelination as some axons had well-developed sheaths while others had only thin myelin coats. Only the group with allografts without either pretreated grafts or host immunosuppression (Group D) had an axonal density ratio of $<1$ although this was not a statistically significant difference (Fig. 4). There were mild inflammatory changes in all nerve grafts as noted below:

\begin{tabular}{cc} 
Group & $\begin{array}{c}\% \text { area with inflammatory } \\
\text { cells/normal nerve }\end{array}$ \\
\hline A & $7.3 \% \pm 4.7 \%$ \\
B & $5.6 \% \pm 6.2 \%$ \\
C & $8.1 \% \pm 8.0 \%$ \\
D & $9.6 \% \pm 10.9 \%$ \\
E & $6.3 \% \pm 2.4 \%$ \\
F & $3.6 \% \pm 4.3 \%$ \\
G & $15.8 \% \pm 7.4 \%$ \\
H & $3.4 \% \pm 3.8 \%$
\end{tabular}

No significant differences were noted between the different groups.

\section{Serum cyclosporin-A assay}

All animals receiving cyclosporin had satisfactory serum levels which ranged from 650 to $1,020 \mathrm{ng} / \mathrm{ml}$.

\section{DISCUSSION}

Despite the ability to transplant many other cadaveric tissues, nerve transplantation is not performed clinically. Although cyclosporin-A has greater efficacy when compared to azathioprine and corticosteroids, it also has greater systemic toxicity. The results of this study demonstrate that animals receiving predegenerated grafts had smaller hind limb flexion contractures and lower hydroxproline ratios of the tibialis anterior muscle as compared to the values obtained from animals without either cyclosporin-A or pretreatment. However, the animals with immunosuppression had greater neurologic recovery than did the animals with predegenerated grafts and no immunosuppression. There were no significant differences between the isografted groups with cyclosporin or without, indicating that cyclosporin itself does not have an inhibitory characteristic on post-surgical inflammation and fibrosis of muscle tissue. All grafted animals demonstrated some neurological recovery when compared to the non-grafted control group. The sensory testing and gait analyses did not appear to be reliable studies in this model that involved grafting a large segment of the sciatic nerve in the animals. The results of the sensory tests were consistent with crossover innervation from the saphenous nerve which has been described previously $/ 34 /$. In their description of the sciatic function index which quantifies the gait analysis, deMedinaceli et al. /29/ were unable to note significant return of foot intrinsic muscles, even after simple transsection repair of the sciatic nerve, although they noted excellent functional recovery following lesions in continuity such as a crush injury. Bain and MacKinnon /36/ did report success using a modification of deMedinaceli's technique. In a careful evaluation of this technique, Terzis and Smith /30/ noted that the return of the function of the intrinsic muscles in the foot was the only reliable portion of the sciatic function index. None of the animals in our study obtained return of function of the foot intrinsic muscles. The animals had ample time for nerve regeneration to the level of the foot based on the site of the most proximal portion of the lesion. In preliminary studies with observation for up to six months, no recovery of the foot intrinsic muscles was noted when large grafts were placed in an intercalary fashion in the sciatic nerve. The evaluation of the hind limb flexion contractures may have been a more sensitive indicator because these contractures are time-dependent. Animals that have a faster recovery will probably have a smaller hind limb flexion contracture even though two groups of animals ultimately have similar numbers of axons reinnervating the end organs. This may explain why the hind limb flexion contractures and the hydroxyproline concentrations (a measure of fibrosis of muscle tissue) demonstrated signifi- 
cant differences, whereas the studies of muscle weights and axonal counts did not. From the histologic studies it appears that the rejection of intercalary grafts is not as dramatic as originally proposed $/ 12,13,15,16 /$. This again may be related to the fact that the differences between the groups were based on the speed of reinnervation, not the ultimate quantity of regeneration of the axons reaching the end organs. Grafts with less immunogenicity or with host immunosup- pression may maintain larger numbers of Schwann cells that facilitate faster regeneration but ultimately the grafts allow similar numbers of axons to reach the motor and sensory end organs. This is highlighted by the fact that there were not significant differences in the inflammatory cell migration into the grafts between the different groups.

The mechanism by which Wallerian degeneration could affect the immunogenicity of the

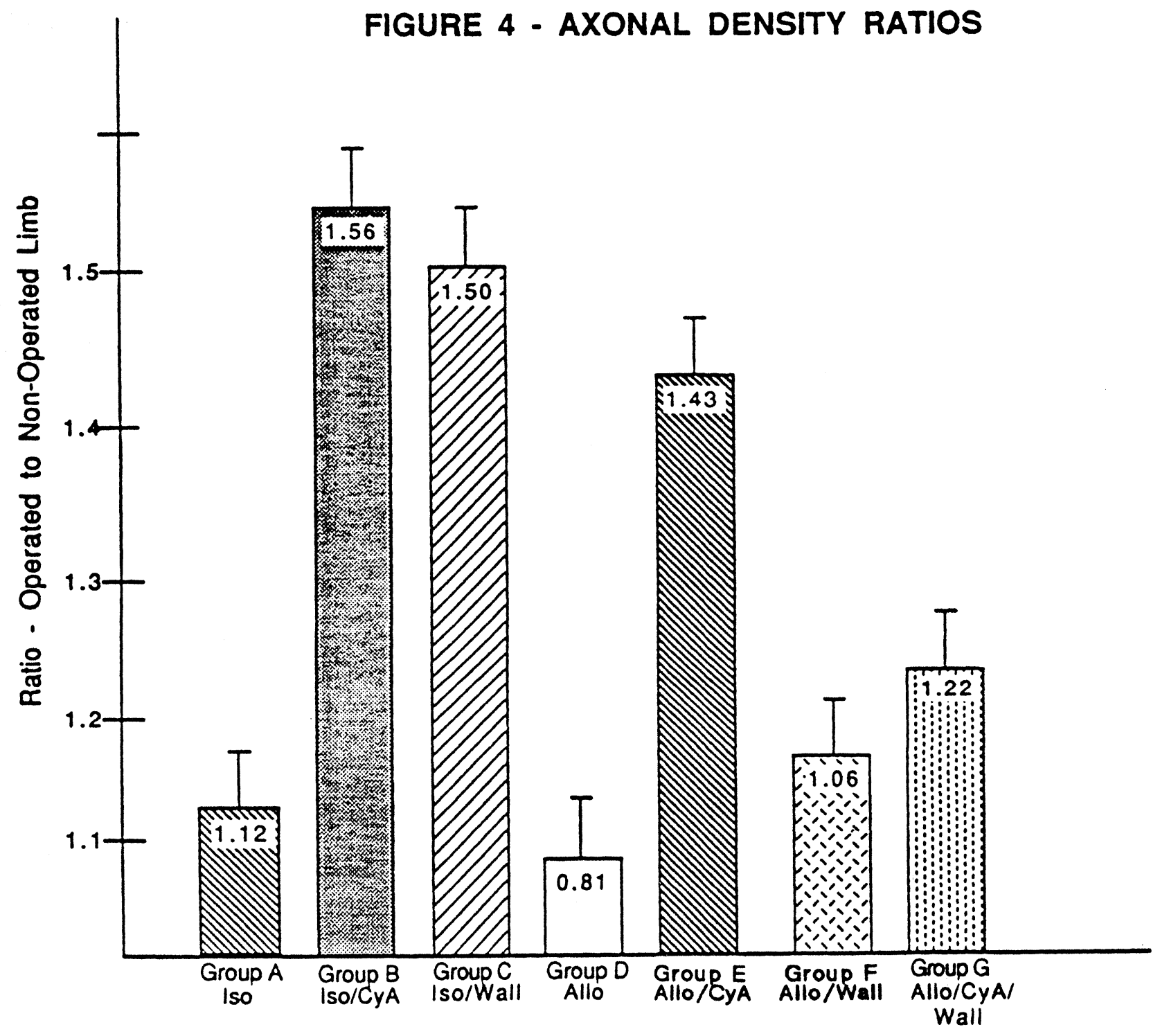

Fig. 4: Axonal density ratios. The axonal density ratio in the nerve segments are expressed as the ratio of the axonal density in the mid portion of the nerve segment of the operated limb compared to the corresponding segment of the sciatic nerve in the non-operated control limb. 
grafts is two-fold. On one hand, the histocompatibility antigens may be less accessible or not expressed as strongly during the process of Wallerian degeneration and conversion of Schwann cells to become phagocytic cells. This may occur for either Class I or Class II antigens. In the evaluation of the effect of trauma on major histocompatibility Class II antigens, an increase in the expression of the antigens was noted following nerve division and Wallerian degeneration /37/. Presumably these antigens which are increased in their expression are also available for recognition by the host. This places some doubt on the importance of MHC Class II antigens in the recognition of allogeneic peripheral nerve tissue. The effect of trauma on the expression of major histocompatibility Class I antigens has not been demonstrated.

On the other hand, the predegenerated graft may allow for more rapid replacement of donor Schwann cells by host Schwann cells. Once this process is completed, the graft would then be rendered non-immunogenic. There is very little information on the use of predegenerated grafts. Gordon et al. /38/ demonstrated an improvement in chronic nerve injuries which were treated with predegenerated syngeneic grafts but not in acute injuries. Predegenerated grafts do not appear to improve neurologic recovery in this study of an acute segmental loss of the nerve by providing activated Schwann cells that enhance overall regeneration as the pretreated grafts did not improve the regeneration following syngeneic transplantation. The use of predegenerated grafts does have the added complexity of requiring donors who have had prior nerve injuries or from living donors.

\section{ACKNOWLEDGEMENTS}

This study was supported by research grants from the Orthopaedic Research and Education Foundation and the American Society for Surgery of the Hand.

This work was presented at the Annual Meeting of the Orthopaedic Research Society, New Orleans, Louisiana, February 1990.

\section{REFERENCES}

1. Albert E. Nerve transplantation. Weiner Med Presse 1885; 26: 1285 .

2. Eden R. Die freie Transplantation der peripheren Neruen sum ersats von Neruendefekten. Arch J Klin Chir 1919; 112: 471-512.

3. Mayo-Robson AW. Nerve grafting as a means of restoring function in limbs paralyzed by gunshot or other injuries. Br Med J 1917; 1: 117-118.

4. Marmor L. Regeneration of peripheral nerves by irradiated homografts. J Bone and Joint Surg 1964; 46A: 383-394.

5. Cajal RY. Degeneration and Regeneration of the Nervous System. Oxford: Oxford Press, 1928.

6. Marmor L. Peripheral Nerve Regeneration Using Nerve Grafts. Springfield, IL: Charles C Thomas, 1967.

7. Marmor L, Miner R, Foster J. Experimental prevention of nerve homograft rejection by use of immunosuppressive drugs. J Neurosurg 1967; 27: 415418.

8. Ducker TB, Hayes GJ. Peripheral nerve grafts: experimental studies in the dog and chimpanzee to define homograft limitations. J Neurosurg 1970; 32: 236-243.

9. Mackinnon SE, Hudson AR, Bain JR, Hunter DA. The peripheral nerve allograft: an assessment of regeneration in the immunosuppressed host. Plast Reconstr Surg 1987; 79: 436.

10. Mackinnon SE, Hudson AR, Bilao J, Kline D. Nerve allograft response: a quantitative immunological study. Neurosurgery 1982; 10: 61-84.

11. Mackinnon SE, Hudson AR, Flak RE, Kline D, Hunter D. Peripheral nerve allograft: an immunological assessment of pretreatment methods. Neurosurgery $1984 ; 14: 167-171$.

12. Zalewski AA, Gulati AK. Rejection of nerve allografts after cessation of immunosuppression with cyclosporin. Transplantation 1981; 31: 88-89.

13. Zalewski AA, Gulati AK. Survival of nerve and Schwann cells in allografts after cyclosporin-A treatment. Exp Neurology 1980; 70: 219-225.

14. Zalewski AA, Gulati AK. Evaluation of histocompatibility as a factor in the repair of nerve with a frozen nerve allograft. J Neurosurg 1982; 56: 550-554.

15. Zalewski AA, Gulati AK. Failure of cyclosporin-A to induce immunological unresponsiveness to nerve allografts. Exp Neurology 1984; 83: 659-663.

16. Zalewski AA, Gulati AK. Survival of nerve allografts in sensitized rats treated with cyclosporin-A. J Neurosurg 1984; 60: 828-834.

17. Zalewski AA, Silvers WK. An evaluation of nerve repair with nerve allografts in normal and immunologically tolerant rats. J Neurosurg 1980; 52: 557-563. 
18. Pollard JD, McLeon JG, Gye, RS. Regeneration through peripheral nerve allografts: an electrophysiological and histological study following the use of immunosuppressive therapy. Arch Neurol 1973; 28: 31-37.

19. Pollard JD, McLeon JG. Fresh and predegenerated nerve allografts and isografts in tremble mice. Muscle and Nerve $1981 ; 4: 274-281$.

20. Mackinnon SE, Hudson AR, Falk RE, Kline D, Hunter D. Peripheral nerve allograft: an assessment of regeneration across pretreated nerve allografts. Neurosurgery 1984; 15: 690-693.

21. Pollard JD, McLeon JG. Nerve grafts in the tremble mouse: an electrophysiological and histological study. J Neurol Sci 1980; 46: 373-383.

22. Singh R. Experience with allografts in the surgery of peripheral nerves. Acta Neurochir 1976; 34: 195-201.

23. Singh R, Mechelse K, Stefanko S. Role of tissue typing on preserved nerve allografts in dogs. J Neurol Neurosurg Psychiatry 1977; 40: 865-871.

24. Singh R, Vriesendorp HM, Mechelse K, Stefanko S. Cadaver nerve allografts in dogs. Biomedicine 1981; 35: 67-70.

25. Almquist E, Eeg-Olofsson G. Sensory-nerveconduction velocity and two-point discrimination in sutured nerves. J Bone and Joint Surg 1970; 52A: 791796.

26. Bolesta MJ, Garrett WE, Ribbeck BM, Glisson RR, Seaber AV, Goldner JL. Immediate and delayed neurorrhaphy in a rabbit model: a functional, histologic and biochemical comparison. J Hand Surg 1988; 13A: 352-357.

27. Palm J, Black G. Interrelationships of inbred rat strains with respect to Ag-B and non-Ag-B antigens. Transplantation 1971; 11: 184-189.

28. Bain J, Mackinnon S, Hudson A. The peripheral nerve allograft: a dose response curve in the rat immunosuppressed with cyclosporin-A. Plast Reconstr
Surg 1988; 82: 447.

29. DeMedinaceli L, Freed WJ, Wyatt RJ. An index of the functional condition of rat sciatic nerve based on measurements made for walking tracks. Exp Neurol 1982; 77: 634-643.

30. Terzis JK, Smith KJ. DeMedinaceli versus microstructure: a critical appraisal of nerve repair. Pres. 2nd Annual Meeting of the American Society for Reconstructive Microsurgery. New Orleans, LA, 1986.

31. Ruwe PA, Lee KE, Trumble TE. A functional evaluation of cryopreserved peripheral nerve autografts. Trans ORS 1988; 13: 465.

32. Woessner JF. The determination of hydroxyproline in tissue and protein samples containing small proportions of this amino acid. Arch Biochem Biophys 1964; 93: 440-447.

33. MacDonald BM, Bolesta MJ, Seaber AV, Garrett WE. Hydroxyproline quantitation in normal muscle and denervated muscle after immediate and delayed nerve repair. Trans ORS 1986; 11: 186.

34. Devor M, Schonfeld D, Seltzer Z, Wall PD. Two modes of cutaneous reinnervation following peripheral nerve injury. J Comp Neur 1979; 185: 211-220.

35. Trumble TE, Whalen JT, Troiano $N$, Jacobsen $S$. Cryoprotection: the effects on cultured Schwann cells and with cryosurgery. Trans ORS 1990; 15: 581.

36. Bain JR, MacKinnon SE. The peripheral nerve allograft: an assessment of regeneration across nerve allografts in rats immunosuppressed with cyclosporinA. Plast Reconstr Surg 1988; 82: 1052-1064.

37. Trumble TE, Stanislaw J, Jacobsen S, Troiano N. Changes in peripheral nerve MHC Class II antigens following trauma. Abstracts for Society of Neuroscience 1990: 15: 1370.

38. Gordon L, Bunke H, Jeweet DL, Muldroney B, Bunge $G$. Predegenerated nerve autografts as compared with fresh nerve autografts in freshly cut and pre-cut motor nerve defects in the rat. J Hand Surg 1979; 4: 42-47. 

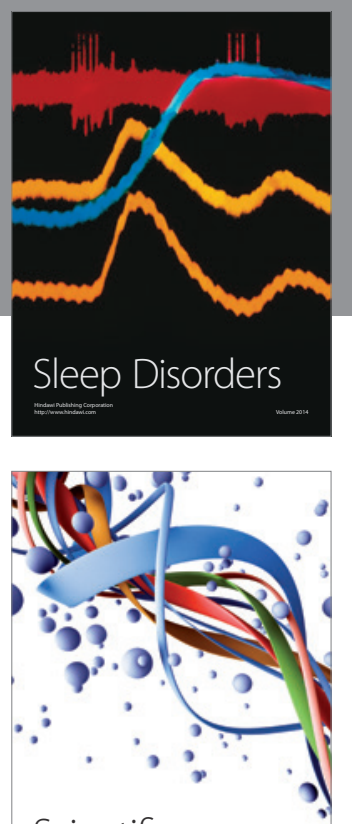

Scientifica
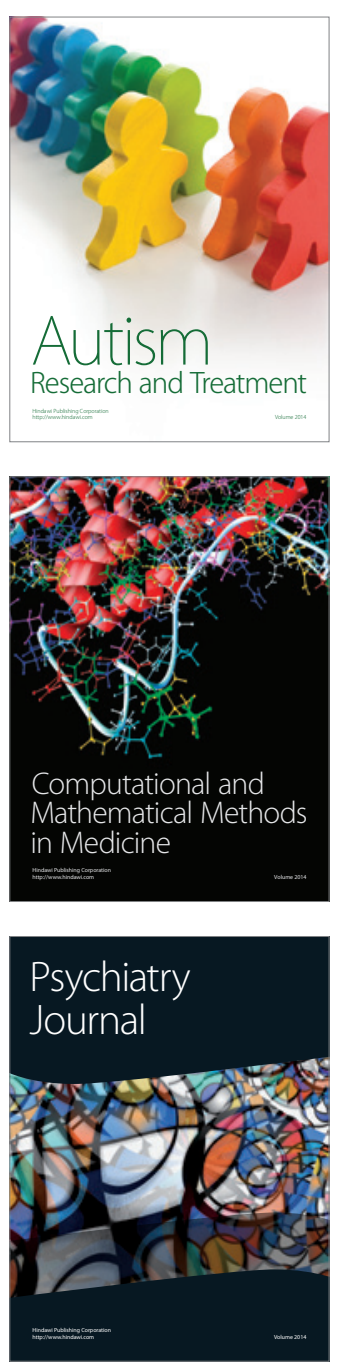
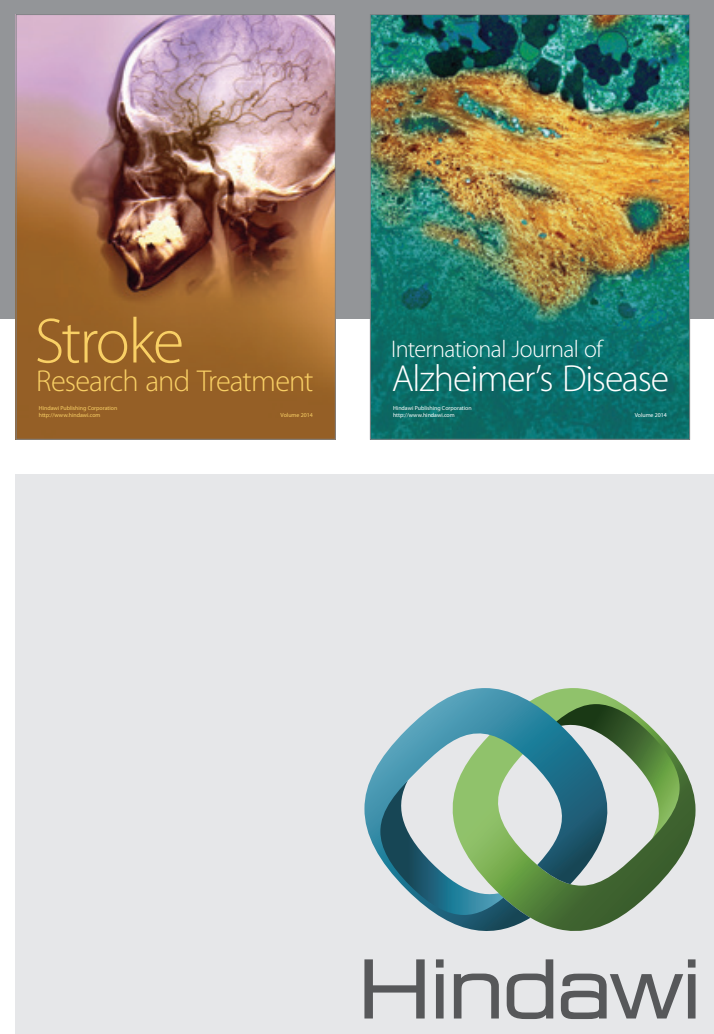

Submit your manuscripts at

http://www.hindawi.com
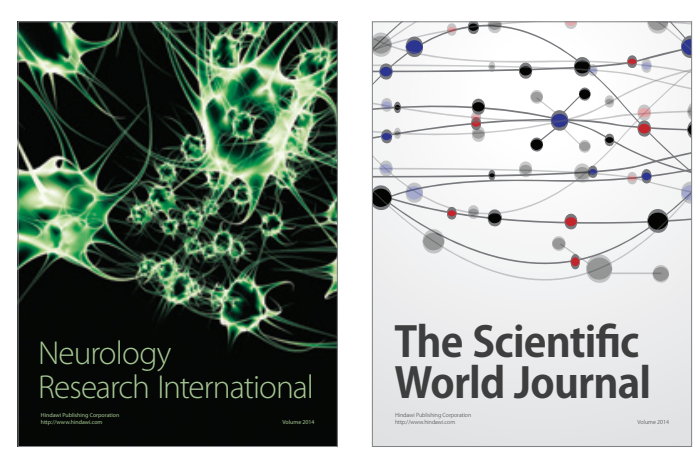

The Scientific World Journal

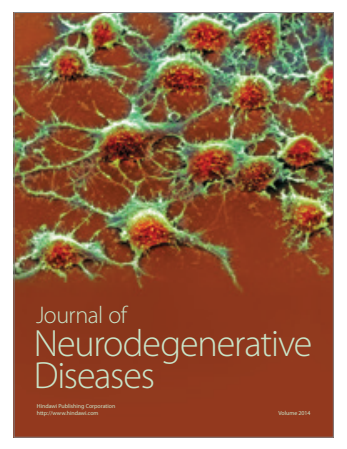

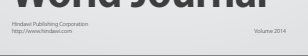

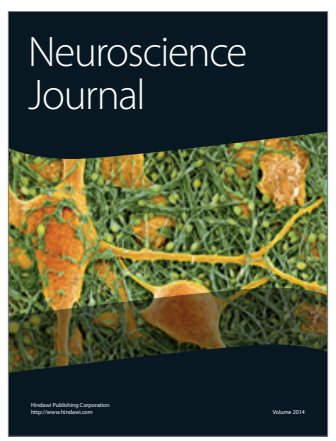

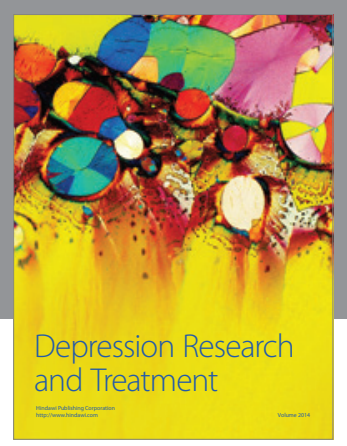
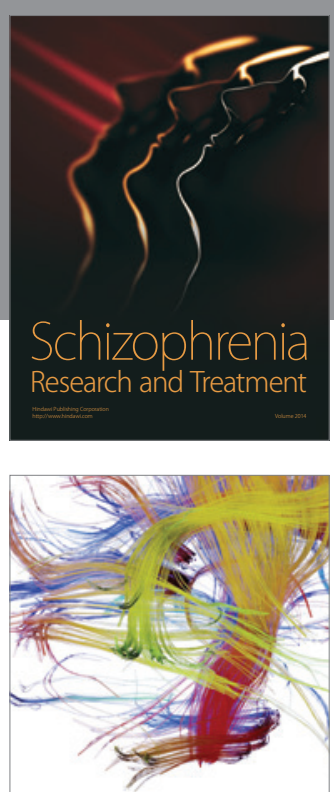

Brain Science

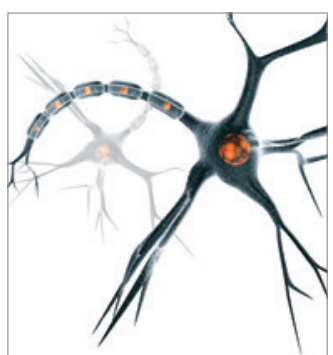

Neural Plasticity
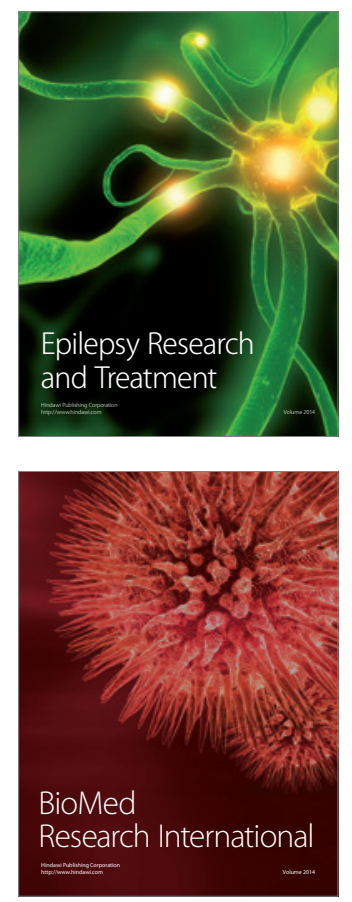

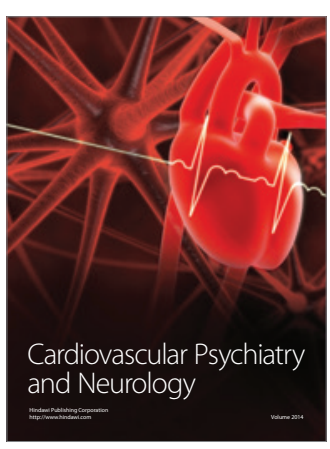

Parkinson's

Disease
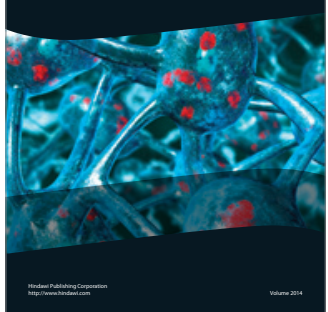